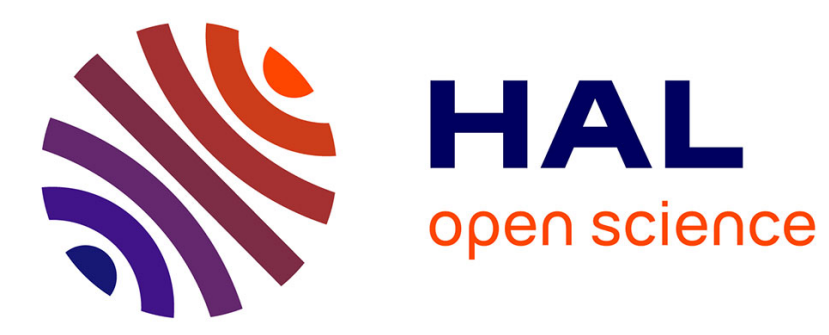

\title{
Mismatch of conductivity anisotropy in the mixed and normal states of type-II superconductors
}

\author{
A. A. Bespalov, A. S. Mel’Nikov
}

\section{To cite this version:}

A. A. Bespalov, A. S. Mel'Nikov. Mismatch of conductivity anisotropy in the mixed and normal states of type-II superconductors. Physical Review B: Condensed Matter and Materials Physics (1998-2015), 2012, 85 (17), pp.1745002 (1-10). 10.1103/PhysRevB.85.174502 . hal-00731814

\section{HAL Id: hal-00731814 \\ https://hal.science/hal-00731814}

Submitted on 13 Mar 2018

HAL is a multi-disciplinary open access archive for the deposit and dissemination of scientific research documents, whether they are published or not. The documents may come from teaching and research institutions in France or abroad, or from public or private research centers.
L'archive ouverte pluridisciplinaire HAL, est destinée au dépôt et à la diffusion de documents scientifiques de niveau recherche, publiés ou non, émanant des établissements d'enseignement et de recherche français ou étrangers, des laboratoires publics ou privés. 


\title{
Mismatch of conductivity anisotropy in the mixed and normal states of type-II superconductors
}

\author{
A. A. Bespalov ${ }^{1,2}$ and A. S. Mel'nikov ${ }^{1}$ \\ ${ }^{1}$ Institute for Physics of Microstructures, Russian Academy of Sciences, GSP-105, 603950, Nizhny Novgorod, Russia \\ ${ }^{2}$ Univ. Bordeaux, LOMA, UMR 5798, F-33600 Talence, France
}

(Received 10 February 2012; published 3 May 2012)

\begin{abstract}
We have calculated the Bardeen-Stephen contribution to the vortex viscosity for uniaxial anisotropic superconductors within the time-dependent Ginzburg-Landau (TDGL) theory. We focus our attention on superconductors with a mismatch of anisotropy of normal and superconducting characteristics. Exact asymptotics for the Bardeen-Stephen contribution have been derived in two limits: (i) $l_{E a b} \ll \xi_{a b}, l_{E c} \ll \xi_{c}$ and (ii) $l_{E c} \gg \xi_{c}$, $l_{E a b} \lesssim \xi_{a b}$, where $l_{E a b}, l_{E c}$ and $\xi_{a b}, \xi_{c}$ are the electric field penetration lengths and the coherence lengths in the $a b$ plane and in the direction of the $c$ axis. Also, we suggest a variational procedure which allows us to calculate the vortex viscosity for superconductors with arbitrary parameters $\xi$ and $l_{E}$. The approximate analytical result is compared with numerical calculations. Finally, using a generalized TDGL theory, we prove that the viscosity anisotropy and, thus, the flux-flow conductivity anisotropy may depend on temperature.
\end{abstract}

DOI: 10.1103/PhysRevB.85.174502

PACS number(s): 74.25.fc, 74.20.De, 74.25.Op, 74.40.Gh

\section{INTRODUCTION}

The existence of a nonzero electrical resistivity in type-II superconductors in the mixed state is connected with the motion of magnetic flux vortices. It can be observed in the presence of a sufficiently large transport current so that pinning is suppressed. In the stationary flux-flow regime, the Lorentz force acting on an isolated vortex is balanced by the intrinsic viscous drag force:

$$
\frac{\phi_{0}}{c}\left(\mathbf{j}_{\mathrm{tr}} \times \mathbf{n}\right)=\eta \mathbf{V}_{L}
$$

Here, $\phi_{0}$ is the flux quantum, $\mathbf{j}_{\text {tr }}$ is the transport current density, $\mathbf{n}$ is the unit vector along the magnetic field, $\mathbf{V}_{L}$ is the vortex velocity, and $\eta$ is a viscous drag coefficient. As vortices move, the magnetic field in the sample becomes nonstationary and a macroscopic electrical field $\mathbf{E}$ is induced, which is connected with the transport current via Ohm's law: $\mathbf{E}=\mathbf{j}_{\mathrm{tr}} / \sigma$. For weak average magnetic fields $B \ll H_{c 2}$, where $H_{c 2}$ is the upper critical field, the flux-flow conductivity $\sigma$ is

$$
\sigma=\frac{c^{2} \eta}{B \phi_{0}} \text {. }
$$

The presence of a finite conductivity implies that current flow is accompanied by dissipation. It has been shown that there are two main mechanisms of dissipation: losses due to relaxation of the order parameter ${ }^{1}$ and Ohmic losses associated with normal currents flowing through the vortex core. ${ }^{2}$

For an anisotropic superconductor, Eq. (2) is generalized as follows:

$$
\hat{\sigma}=\frac{c^{2}}{B \phi_{0}}\left(\begin{array}{cc}
\eta_{y y} & -\eta_{y x} \\
-\eta_{x y} & \eta_{x x}
\end{array}\right)
$$

with the $z$ axis along the magnetic field. The peculiar structure of the conductivity tensor is explained by the fact that the $x$ component of the electric field depends on the $y$ component of the vortex velocity, and vice versa.

It can be seen from Eq. (3) that the flux-flow conductivity is determined by the magnetic field and the viscosity tensor $\hat{\eta}$. A rigorous approach to the problem of viscosity evaluation has been first suggested by $\mathrm{Schmid}^{3}$ and was later developed by Gor'kov and Kopnin ${ }^{4}$ (see also Ref. 5 for review). Their method is based on the time-dependent Ginzburg-Landau (TDGL) theory. Within this model, the flux-flow conductivity has been evaluated for isotropic superconductors in several papers. ${ }^{6-8}$ Both viscosity components due to order-parameter relaxation and Ohmic losses (frequently called the BardeenStephen contribution) have been derived.

Theoretical studies of free flux flow in anisotropic materials have been stimulated by the discovery of high-temperature superconductors, which appeared to possess rather strong anisotropy. A number of papers have addressed this problem using different models and approximations. ${ }^{9-11}$ The procedure of viscosity calculation can be essentially simplified in the limit of dirty uniaxial superconductors with the ratio $s_{0}=$ $m_{c} \sigma_{c} / m_{a b} \sigma_{a b}$ equal to unity. Here, $\sigma_{c}, \sigma_{a b}$ and $m_{c}, m_{a b}$ are the normal conductivities and Cooper-pair masses in the direction of the anisotropy axis $c$ and in the perpendicular $a b$ plane, respectively. The condition $s_{0}=1$ allows us to reduce the problem of anisotropic vortex dynamics to an isotropic one by means of a scaling transformation. ${ }^{10}$ Yet, this is not true in the case $s_{0} \neq 1$, i.e, for a mismatch of anisotropies of Cooper-pair masses and normal conductivities. Such a mismatch is theoretically possible in the relatively clean limit ${ }^{12}$ and it may have been experimentally observed in a new class of Fe-based pnictide superconductors. According to Ref. 13, the ratio $\sigma_{a b} / \sigma_{c}$ in $\mathrm{PrFeAsO}_{0.7}$ is close to 120 , whereas $m_{c} / m_{a b}$ in the same compound is about 25 , as determined in Ref. 14 from upper critical field measurements. In Refs. 15 and 16, anisotropies of the same order in $\mathrm{Ba}_{1-x} \mathrm{~K}_{x} \mathrm{Fe}_{2} \mathrm{As}_{2}$ are reported. However, existing experimental data for the pnictides are contradictory. In Ref. 17, a relatively low-resistivity anisotropy in $\mathrm{BaFe}_{2} \mathrm{As}_{2}$ is given: $\sigma_{a b} / \sigma_{c} \sim 2-3$. In some works, ${ }^{14,18}$ an anisotropy mismatch has not been clearly detected. Previous calculations of the viscous drag tensor accounted for the anisotropy mismatch only on the basis of a simplified model of a steplike order-parameter profile within the vortex core. ${ }^{9}$ Of course, a detailed comparison with experimental data demands these calculations to be generalized for a more realistic order-parameter profile.

In this paper, we evaluate analytically the viscosity tensor for a realistic gap profile within the core, focusing our attention 
on the case $s_{0} \neq 1$ and considering both standard TDGL model for gapless superconductors and its generalization for superconductors with a finite gap. ${ }^{12,19}$ Note that for Fe-based materials, the gapless regime may be achievable due to the strong interband scattering on dopant's ions. ${ }^{20}$ In Sec. II, we derive the basic equations following the approach of Gor'kov and Kopnin. ${ }^{5}$ In Sec. III, we develop approximate methods based on different assumptions about the ratio of the electric field penetration depth to the coherence length. The results of preceding works ${ }^{6,7,9}$ are revised and improved. In the end of this section, we consider a variational principle, which provides us with a simple general relation for the BardeenStephen contribution. In Sec. IV, the problem is considered in the framework of a generalized TDGL theory. Here, we derive our main result: we predict that the flux-flow conductivity anisotropy may depend on temperature in superconductors with the parameter $s_{0} \neq 1$.

\section{BASIC EQUATIONS}

Following Gor'kov and Kopnin, ${ }^{5}$ we start the analysis of vortex motion with the TDGL equation for the superconducting order parameter $\psi$ :

$$
\begin{gathered}
\gamma\left(\hbar \frac{\partial \psi}{\partial t}+2 i e \Phi \psi\right)=-\frac{\delta F}{\delta \psi^{*}} \\
F=\int\left[\left(i \hbar \nabla-\frac{2 e}{c} \mathbf{A}\right) \psi^{*} \frac{\hat{m}^{-1}}{2}\left(-i \hbar \nabla-\frac{2 e}{c} \mathbf{A}\right) \psi\right. \\
\left.+a|\psi|^{2}+\frac{1}{2} b|\psi|^{4}\right] d^{3} \mathbf{r} .
\end{gathered}
$$

Here, $F$ is the usual GL free energy, $\gamma$ is a relaxation constant, and $\mathbf{A}$ and $\Phi$ are the vector and scalar potentials, respectively. We consider uniaxial anisotropic superconductors, so the Cooper-pair mass $\hat{m}$ is a tensor with components $m_{i j}=$ $m_{a b}\left(\delta_{i j}+\mu v_{i} v_{j}\right)$, where $\boldsymbol{v}$ is the unit vector along the $c$ axis, $\mu=m_{c} / m_{a b}-1$. Equation (4) is supplemented by the equation for the current density

$$
\operatorname{div} \mathbf{j}=0
$$

where

$\mathbf{j}=2 e|\psi|^{2} \hat{m}^{-1}\left(\hbar \nabla \theta-\frac{2 e}{c} \mathbf{A}\right)-\hat{\sigma}_{n}\left(\nabla \Phi+\frac{1}{c} \frac{\partial \mathbf{A}}{\partial t}\right)$.

Here, $\theta=\arg (\psi)$ and $\hat{\sigma}_{n}$ is the normal-state conductivity tensor with components $\sigma_{n i j}=\sigma_{a b} \delta_{i j}+\left(\sigma_{c}-\sigma_{a b}\right) v_{i} v_{j}$. For simplicity, we will consider only superconductors with a large Ginzburg-Landau parameter $\kappa=\lambda / \xi \gg 1$, where $\lambda$ is the London penetration length and $\xi$ is the superconducting coherence length. One can prove that $|2 e \mathbf{A} / c| /|\hbar \nabla \theta| \ll 1$ at distances much smaller than $\lambda$ from the vortex axis in the gauge where $\mathbf{A}=0$ on the vortex axis and $\operatorname{div} \mathbf{A}=0$. Imposing the additional condition $l_{E}^{2} / \lambda \xi \ll 1$, where $l_{E}$ is the electric field penetration depth [see Eq. (12)], one can neglect the term

in Eq. (6).

$$
\frac{1}{c} \frac{\partial \mathbf{A}}{\partial t}
$$

Let us consider the orientation of the internal magnetic field at angle $\varphi$ to the crystallographic $c$ axis. We choose

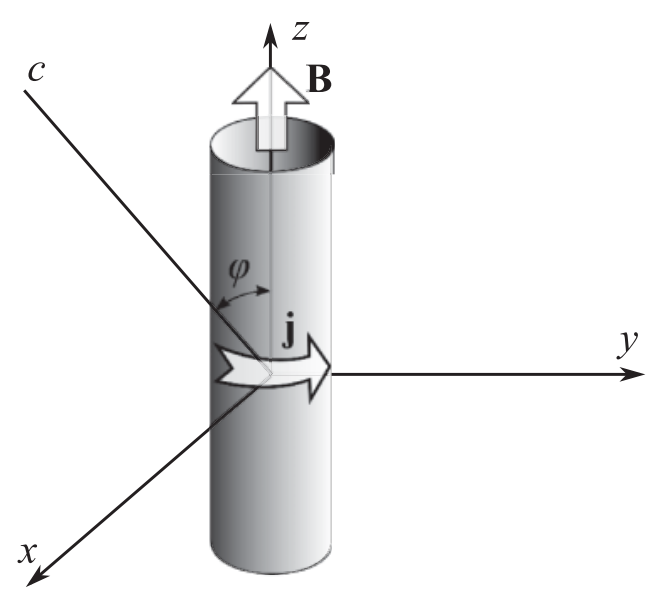

FIG. 1. The coordinate frame.

the coordinate frame $(x, y, z)$ with the $z$ axis coinciding with the vortex axis and with the $c$ axis lying in the $x z$ plane (see Fig. 1). In this frame, the functions $\psi$ and $\Phi$ do not depend on $z$, and the tensor $\hat{\eta}$ is diagonal.

The derivation of the force balance equation (1) and the explicit expression for the viscous drag tensor may be found in Refs. 5 and 9. However, in Appendix A, we outline the calculations for the reader's convenience.

The components of the Bardeen-Stephen contribution $\hat{\eta}_{\mathrm{oh}}$ are given by

$$
\begin{aligned}
& \eta_{x}=-2 \frac{|a|}{b} \gamma \hbar \int f^{2}\left(\rho_{1}\right) \frac{y_{1}}{\rho_{1}^{2}}\left(u^{2} \Phi_{x}-\frac{y_{1}}{\rho_{1}^{2}}\right) d x_{1} d y_{1}, \\
& \eta_{y}=-2 \frac{|a|}{b} \gamma \hbar \int f^{2}\left(\rho_{1}\right) \frac{x_{1}}{\rho_{1}^{2}}\left(u^{2} \Phi_{y}-\frac{x_{1}}{\rho_{1}^{2}}\right) d x_{1} d y_{1} .
\end{aligned}
$$

Here,

$$
\begin{gathered}
\eta_{x}=\left[m(\varphi) / m_{a b}\right]^{1 / 2}\left(\eta_{\mathrm{oh}}\right)_{x x}, \\
\eta_{y}=\left[m_{a b} / m(\varphi)\right]^{1 / 2}\left(\eta_{\mathrm{oh}}\right)_{y y}, \\
m(\varphi)=\frac{m_{a b}(1+\mu)}{1+\mu \cos ^{2} \varphi}, \quad u=\xi_{a b} / l_{E a b}, \\
\left(x_{1}, y_{1}\right)=\xi_{a b}^{-1}\left(\sqrt{\frac{m(\varphi)}{m_{a b}}} x, y\right), \quad \rho_{1}=\sqrt{x_{1}^{2}+y_{1}^{2}},
\end{gathered}
$$

$\xi_{a b}$ and $l_{E a b}$ are the coherence length and the electric field penetration depth in the $a b$ plane, respectively:

$$
\xi_{a b}=\sqrt{\frac{\hbar^{2}}{2 m_{a b}|a|}}, \quad l_{E a b}=\left[\hbar \sigma_{a b} /\left(8 e^{2} \gamma \frac{|a|}{b}\right)\right]^{1 / 2} .
$$

The function $f(\rho)$ describes the profile of the dimensionless order-parameter modulus in a static isotropic vortex. This function satisfies the relation

$$
\frac{1}{\rho} \frac{d}{d \rho}\left(\rho \frac{d f}{d \rho}\right)-\frac{f}{\rho^{2}}+f-f^{3}=0,
$$

which follows from Eqs. (A8) and (A17). The boundary conditions are $f(0)=0, f(\infty)=1$. The functions $\Phi_{x}$ and 
$\Phi_{y}$ in Eqs. (7) and (8) should be determined from the linear equations

$$
\begin{aligned}
& s \frac{\partial^{2} \Phi_{x}}{\partial x_{1}^{2}}+\frac{\partial^{2} \Phi_{x}}{\partial y_{1}^{2}}=\left(u^{2} \Phi_{x}-\frac{y_{1}}{\rho_{1}^{2}}\right) f^{2}\left(\rho_{1}\right), \\
& s \frac{\partial^{2} \Phi_{y}}{\partial x_{1}^{2}}+\frac{\partial^{2} \Phi_{y}}{\partial y_{1}^{2}}=\left(u^{2} \Phi_{y}-\frac{x_{1}}{\rho_{1}^{2}}\right) f^{2}\left(\rho_{1}\right),
\end{aligned}
$$

where

$$
s(\varphi)=1+\left(\frac{m_{c} \sigma_{c}}{m_{a b} \sigma_{a b}}-1\right) \frac{\sin ^{2} \varphi}{1+\mu \cos ^{2} \varphi}>0 .
$$

The electric potential can be expressed in terms of $\Phi_{x}$ and $\Phi_{y}$ via

$$
\Phi=\left(\Phi_{x} \sqrt{\frac{m(\varphi)}{m_{a b}}} V_{L x}-\Phi_{y} V_{L y}\right) \frac{4 \gamma e \hbar}{b \sigma_{a b}} \sqrt{\frac{|a|}{2 m_{a b}}} .
$$

Note that there is a relation connecting the components $\eta_{x}$ and $\eta_{y}$ :

$$
\eta_{y}(s, u)=\eta_{x}\left(\frac{1}{s}, \frac{u}{\sqrt{s}}\right) .
$$

In the next section, we consider some limiting cases.

\section{APPROXIMATE SOLUTIONS}

\section{A. The $l_{E} \ll \xi$ limit}

Consider such materials that the electric field penetration length is much smaller than the coherence length:

$$
l_{E a b} \ll \xi_{a b}, \quad l_{E c} \ll \xi_{c} .
$$

This limiting case is closer to gapless superconductors with a high concentration of magnetic impurities, which are characterized by the ration $\xi / l_{E}=\sqrt{12}$. The conditions (19) impose the following restrictions on the parameters $s$ and $u: u \gg 1, s \ll u^{2}$. In this section, we will analyze the case $s \lesssim 1$. The case $1 \ll s \ll u^{2}$ can be considered in a similar way by dividing Eqs. (14) and (15) by $s$. We shall search the asymptotics of the viscosity when $u \rightarrow \infty$ neglecting small terms of order higher than $u^{-2}$ (however, it will be shown that one should keep terms of the order of $u^{-2}$ ).

Our approximation is based on the fact that the characteristic length scale for the functions $\Phi_{x}$ and $\Phi_{y}$ is $u^{-1}$. Hence, the unknown functions reach their asymptotic behavior at distances $\rho \ll 1$ from the vortex axis, where the orderparameter profile $f(\rho)$ is well approximated by the first several terms of its Taylor series:

$$
f^{2}(\rho) \approx k_{2} \rho^{2}+k_{4} \rho^{4}+k_{6} \rho^{6} .
$$

We substitute this expansion into Eq. (14) and introduce the new variables $\tilde{\rho}=\rho_{1} \sqrt{u}, \tilde{\Phi}_{x}=\Phi_{x} u^{3 / 2}$ :

$$
\begin{aligned}
& \frac{\partial^{2} \tilde{\Phi}_{x}}{\partial \tilde{y}^{2}}+s \frac{\partial^{2} \tilde{\Phi}_{x}}{\partial \tilde{x}^{2}} \\
& \quad=\left(k_{2} \tilde{\rho}^{2}+k_{4} \frac{\tilde{\rho}^{4}}{u}+\ldots\right)\left(\tilde{\Phi}_{x}-\frac{\tilde{y}}{\tilde{\rho}^{2}}\right) .
\end{aligned}
$$

Further, the tilde over $\tilde{x}$ and $\tilde{y}$ will be omitted. The solution of Eq. (20) can be expanded in the powers of $u^{-1}$ :

$$
\tilde{\Phi}_{x}=\Phi_{x}^{(0)}+u^{-1} \Phi_{x}^{(1)}+R_{x},
$$

where $\Phi_{x}^{(0)}$ and $\Phi_{x}^{(1)}$ satisfy the following relations:

$$
\begin{gathered}
\frac{\partial^{2} \Phi_{x}^{(0)}}{\partial y^{2}}+s \frac{\partial^{2} \Phi_{x}^{(0)}}{\partial x^{2}}=k_{2} \rho^{2} \Phi_{x}^{(0)}-k_{2} y, \\
\frac{\partial^{2} \Phi_{x}^{(1)}}{\partial y^{2}}+s \frac{\partial^{2} \Phi_{x}^{(1)}}{\partial x^{2}}=k_{2} \rho^{2} \Phi_{x}^{(1)}+k_{4} \rho^{4}\left(\Phi_{x}^{(0)}-\frac{y}{\rho^{2}}\right),
\end{gathered}
$$

and $R_{x}$ is a remainder term. It is proved in Appendix $\mathrm{B}$ that an analogous expansion can be made in the integral in the right-hand side of Eq. (7):

$$
\eta_{x}=-2 \frac{|a|}{b} \gamma \hbar\left[\frac{I_{1 x}(s)}{u}+\frac{I_{2 x}(s)}{u^{2}}+o\left(u^{-2}\right)\right],
$$

where

$$
I_{1 x}(s)=\int k_{2} y\left(\Phi_{x}^{(0)}-\frac{y}{\rho^{2}}\right) d x d y,
$$

$I_{2 x}(s)=\int \frac{y}{\rho^{2}}\left[k_{4} \rho^{4}\left(\Phi_{x}^{(0)}-\frac{y}{\rho^{2}}\right)+k_{2} \rho^{2} \Phi_{x}^{(1)}\right] d x d y$.

The viscosity component $\eta_{y}$ can be calculated similarly:

$$
\eta_{y}=-2 \frac{|a|}{b} \gamma \hbar\left[\frac{I_{1 y}(s)}{u}+\frac{I_{2 y}(s)}{u^{2}}+o\left(u^{-2}\right)\right] .
$$

Using Eq. (18), we obtain

$$
I_{1 y}(s)=I_{1 x}\left(s^{-1}\right) \sqrt{s}, \quad I_{2 y}(s)=I_{2 x}\left(s^{-1}\right) s .
$$

In principle, the functions $I_{1 x}(s)$ and $I_{2 x}(s)$ can be determined by numerical calculations; however, in Sec. III C we present some analytical expressions for these functions.

In Ref. 9, the $u \gg 1$ limit was considered using the Bardeen-Stephen model. ${ }^{2}$ This approach is essentially based on the assumption about a steplike order-parameter profile within the core and does not allow us to obtain a leading term of the order of $u^{-1}$ in the expansion (24).

The particular case $s=1$ has been considered in a number of works mentioned above..$^{3,6-8}$ It corresponds to isotropic superconductors, or anisotropic superconductors with no anisotropy mismatch: $\left(m_{c} \sigma_{c}\right) /\left(m_{a b} \sigma_{a b}\right)=1$. If $s=1$, Eqs. (22) and (23) can be solved exactly:

$$
\begin{gathered}
\Phi_{x}^{(0)}=\frac{1-\exp \left(-\sqrt{k_{2}} \rho^{2} / 2\right)}{\rho^{2}} y, \\
\Phi_{x}^{(1)}=\frac{k_{4} y}{u k_{2}}\left(\frac{1}{4}+\frac{\sqrt{k_{2}} \rho^{2}}{8}\right) \exp \left(-\sqrt{k_{2}} \rho^{2} / 2\right) .
\end{gathered}
$$

After some integration, we obtain a simple relation for the viscous drag coefficients:

$$
\begin{gathered}
\eta_{x}=\eta_{y}=2 \pi \frac{|a|}{b} \gamma \hbar \alpha_{2}(u), \\
\alpha_{2}(u) \approx \frac{\sqrt{k_{2}}}{u}+\frac{k_{4}}{2 k_{2} u^{2}}=\frac{0.583}{u}-\frac{1}{8 u^{2}} .
\end{gathered}
$$

Here, the value $\sqrt{k_{2}}=0.583$ was taken from Ref. 7 , and the relation $k_{4}=-k_{2} / 4$ follows from Eq. (13).

It is appropriate to recall here the result obtained by $\mathrm{Hu}^{7}$ :

$$
\alpha_{2}=\frac{\mathrm{K}_{0}(\delta u)}{\delta u \mathrm{~K}_{1}(\delta u)},
$$

where $\mathrm{K}_{0}$ and $\mathrm{K}_{1}$ are the modified Bessel functions of an imaginary $\operatorname{argument}$ and $\delta$ is a fitting parameter. Equation (31) 
was derived from the exact solution of Eq. (A12) with an approximate order-parameter profile ${ }^{3,21}$ :

$$
f(\rho)=\frac{\rho}{\sqrt{\delta^{2}+\rho^{2}}} .
$$

According to $\mathrm{Schmid}^{3}$ and $\mathrm{Hu}^{7}$ the optimal value of $\delta$ is $\sqrt{2}$, which follows from a variational principle. We can compare different values of $\alpha_{2}(u)$. When $u=\sqrt{12}$, Eq. (31) yields $\alpha_{2}=0.186$, Eq. (30) yields $\alpha_{2}=0.158$, while the numerical result is $\alpha_{2}=0.159 .{ }^{8}$ Our formula gives an error less than $1 \%$. If we keep only the term of order $u^{-1}$ in Eq. (30), we will get a $6 \%$ error, which increases with decreasing $u$.

\section{B. The $l_{E c} \gg \xi_{c}$ limit}

Consider the range of parameters $s \gg u^{2}$ and $u \gtrsim 1$. In terms of $l_{E}, \xi$, and $\varphi$, these conditions read as

$$
l_{E c} \gg \xi_{c}, \quad l_{E a b} \lesssim \xi_{a b}, \quad \cos ^{2} \varphi \ll \frac{\sigma_{c} l_{E a b}^{2}}{\sigma_{a b} \xi_{a b}^{2}} .
$$

Thus, the magnetic field must make a small angle with the $a b$ plane.

When $s \gg u^{2}$, the term $u^{2} \Phi_{x}$ in Eq. (14) is negligible compared to $y_{1} / \rho_{1}^{2}$ in the region $\rho \ll \sqrt{s} / u$, so we immediately obtain from Eq. (7)

$$
\eta_{x} \sim \ln s / u^{2} .
$$

More complicated calculations, which can be found in Appendix C, yield

$$
\begin{aligned}
& \eta_{x}=2 \pi \hbar \gamma \frac{|a|}{b}\left(\ln \frac{\sqrt{s}}{u}-1.475\right), \\
& \eta_{y}=2 \pi \hbar \gamma \frac{|a|}{b}\left(\ln \frac{\sqrt{s}}{u}-0.475\right) .
\end{aligned}
$$

Note that in Ref. 9 in the $u \ll 1$ limit, similar expressions containing $\ln u^{-1}$ have been derived. This similarity is not accidental: the presence of the $\operatorname{logarithm} \ln \left(l_{E} / \xi\right)$ is a characteristic feature of the $l_{E} \gg \xi$ limit.

\section{A variational principle}

In this section, we suggest a simple variational procedure for the calculation of the viscous drag tensor. According to Ref. 5, a general expression for the dissipation function $W[\Phi]$ reads as

$$
W[\Phi]=\nabla \Phi \hat{\sigma}_{n} \nabla \Phi+\frac{2 \gamma}{\hbar}\left|\hbar \frac{\partial \psi}{\partial t}+2 i e \Phi \psi\right|^{2} .
$$

The electric potential should be found from Eq. (A12), which can be viewed as a condition of zero variational derivative of the functional

$$
\int_{z=0} W[\Phi] d^{2} \rho
$$

Thus, the minimum of the functional above equals the loss power per unit length of a moving vortex:

$$
\mathbf{V}_{L} \hat{\eta} \mathbf{V}_{L}=\min _{\Phi} \int_{z=0} W[\Phi] d^{2} \rho
$$

This relation allows us to apply the direct variational method to our problem.
For the sake of convenience, we rewrite Eq. (36) in our rescaled coordinate frame separately for both components of the Bardeen-Stephen contribution:

$$
\begin{gathered}
\eta_{x}=\tilde{\eta}(s, 1, u), \quad \eta_{y}=\tilde{\eta}(1, s, u), \\
\tilde{\eta}\left(s_{x}, s_{y}, u\right)=2 \frac{|a|}{b} \gamma \hbar u^{2} \min _{\phi} \int\left[s_{x}\left(\frac{\partial \phi}{\partial x}\right)^{2}\right. \\
\left.+s_{y}\left(\frac{\partial \phi}{\partial y}\right)^{2}+\frac{f^{2}(\rho)}{u^{2}}\left(u^{2} \phi-\frac{y}{\rho^{2}}\right)^{2}\right] d x d y .
\end{gathered}
$$

Equations (37) and (38) have two important consequences. First, the viscous drag tensor is positively defined when $\sigma_{a b}>$ 0 and $\sigma_{c}>0$. Second, the components of $\hat{\eta}_{\mathrm{oh}}$ increase as the conductivity increases:

$$
\frac{\partial \eta_{i}}{\partial \sigma_{a b}}>0, \quad \frac{\partial \eta_{i}}{\partial \sigma_{c}}>0, \quad i=x, y .
$$

We can obtain an upper estimate for the viscosity components if we substitute a trial function into Eq. (38). In order to find an appropriate trial function, consider the exact equation for $\phi$ :

$$
s_{x} \frac{\partial^{2} \phi}{\partial x^{2}}+s_{y} \frac{\partial^{2} \phi}{\partial y^{2}}=\left(u^{2} \phi-\frac{y}{\rho^{2}}\right) f^{2}(\rho) .
$$

The solution of this equation is an even function of $x$ and an odd function of $y$, so its Fourier series has the form

$$
\phi=\sum_{n=0}^{\infty} \phi_{2 n+1}(\rho) \sin (2 n+1) \chi,
$$

where $\chi$ is the polar angle in the $x y$ plane. When $\rho$ is sufficiently large, $\phi \approx y /\left(u^{2} \rho^{2}\right)$, that means that the series in Eq. (40) contains only the first term. Thus, the trial function

$$
\phi_{t}=\frac{4 \tilde{\phi}(\rho)}{s_{x}+3 s_{y}} \sin \chi
$$

has the correct parity and the correct asymptotics. Let us substitute this function into Eq. (38):

$$
\begin{aligned}
\tilde{\eta} \approx & 2 \frac{|a|}{b} \gamma \hbar \pi \tilde{u}^{2} \min _{\tilde{\phi}} \int_{0}^{\infty} \rho\left[\left(\frac{d \tilde{\phi}}{d \rho}\right)^{2}+\frac{\tilde{\phi}^{2}}{\rho^{2}}\right. \\
& \left.+\frac{f^{2}(\rho)}{\tilde{u}^{2}}\left(\tilde{u}^{2} \tilde{\phi}-\frac{1}{\rho}\right)^{2}\right] d \rho,
\end{aligned}
$$

where

$$
\tilde{u}=u\left(\frac{s_{x}}{4}+\frac{3 s_{y}}{4}\right)^{-1 / 2} .
$$

The differential equation for $\tilde{\phi}$ is

$$
-\frac{1}{\rho} \frac{d}{d \rho}\left(\rho \frac{d \tilde{\phi}}{d \rho}\right)+\frac{\tilde{\phi}}{\rho^{2}}+f^{2}(\rho)\left(\tilde{u}^{2} \tilde{\phi}-\frac{1}{\rho}\right)=0 .
$$

Note that we obtain exactly the same equation if we substitute $\Phi_{x}=\tilde{\phi}(\rho) \sin \chi$ into Eq. (14) when $s=1$ and $u=\tilde{u}$. This means that the trial function (41) reduces our problem to an isotropic one. Unfortunately, an exact solution of Eq. (43) 
is unknown. However, $\mathrm{Schmid}^{3}$ found a solution with an approximate order-parameter profile [see Eq. (32)]:

$$
\tilde{\phi}=\frac{\mathrm{K}_{1}(\tilde{u} \delta) \delta-\sqrt{\delta^{2}+\rho^{2}} \mathrm{~K}_{1}\left(\tilde{u} \sqrt{\delta^{2}+\rho^{2}}\right)}{\delta \mathrm{K}_{1}(\tilde{u} \delta) \tilde{u}^{2} \rho} .
$$

By using this function and the expression (32) for $f$, we can calculate the right-hand side of Eq. (42):

$$
\tilde{\eta} \approx 2 \frac{|a|}{b} \gamma \hbar \pi \frac{\mathrm{K}_{0}(\delta \tilde{u})}{\delta \tilde{u} \mathrm{~K}_{1}(\delta \tilde{u})} .
$$

We take $\delta=f^{\prime}(0)^{-1}=k_{2}^{-1 / 2}$ in order to obtain the correct asymptotics when $u \rightarrow \infty, s=1$ [this asymptotics is determined by $f^{\prime}(0)$ (see Sec. III A)]. Finally, combining (37) and (44) we get approximate relations for the components of $\hat{\eta}^{\prime}$ :

$$
\begin{gathered}
\eta_{x} \approx 2 \pi \frac{|a|}{b} \gamma \hbar \frac{f^{\prime}(0)}{2 u} \sqrt{s+3} \frac{\mathrm{K}_{0}\left(\frac{2 u}{f^{\prime}(0) \sqrt{s+3}}\right)}{\mathrm{K}_{1}\left(\frac{2 u}{f^{\prime}(0) \sqrt{s+3}}\right)}, \\
\eta_{y} \approx 2 \pi \frac{|a|}{b} \gamma \hbar \frac{f^{\prime}(0)}{2 u} \sqrt{3 s+1} \frac{\mathrm{K}_{0}\left(\frac{2 u}{f^{\prime}(0) \sqrt{3 s+1}}\right)}{\mathrm{K}_{1}\left(\frac{2 u}{f^{\prime}(0) \sqrt{3 s+1}}\right)} .
\end{gathered}
$$

No restrictions on the parameters $s$ and $u$ are implied here.

Let us check if these relations are in accordance with the results from Secs. III A and III B. Expanding $\eta_{x}$ in the form (45) in the powers of $u^{-1}$ when $u \gg 1$ and $s \lesssim 1$, we obtain the following expressions for the coefficients $I_{1 x}$ and $I_{2 x}$, which were introduced in Sec. III A [see Eq. (24)]:

$$
I_{1 x}(s)=-\frac{\pi \sqrt{k_{2}} \sqrt{s+3}}{2}, \quad I_{2 x}(s)=\frac{\pi k_{2}(s+3)}{8} .
$$

When $s=1$,

$$
\eta_{x}=\eta_{y}=2 \pi \frac{|a|}{b} \gamma \hbar \frac{f^{\prime}(0)}{u}+O\left(u^{-2}\right),
$$

which should be compared with Eq. (30). The perfect agreement between the exact and approximate result is not surprising because the trial function (41) is the exact solution of our variational problem in the isotropic case.

In order to check whether Eqs. (45) and (46) are applicable for $s \neq 1$, we used numerical calculations. We solved Eq. (39) in the region $x>0, y>0$ with the boundary conditions

$$
\left.\frac{\partial \phi}{\partial x}\right|_{x=0}=0,\left.\quad \phi\right|_{y=0}=0
$$

A sufficiently large $450 \times 450$ mesh with a $0.03 \times 0.03$ unit cell has been used. The numerical algorithm applied was the method of steepest descent. After the determination of the function $\phi(\rho)$, numerical integration has been performed.

When $s=0$, Eqs. (47) and (28) give

$$
\begin{aligned}
& \eta_{x}=2 \frac{|a|}{b} \gamma \hbar \frac{1.59}{u}+O\left(u^{-2}\right), \\
& \eta_{y}=2 \frac{|a|}{b} \gamma \hbar \frac{0.92}{u}+O\left(u^{-2}\right) .
\end{aligned}
$$
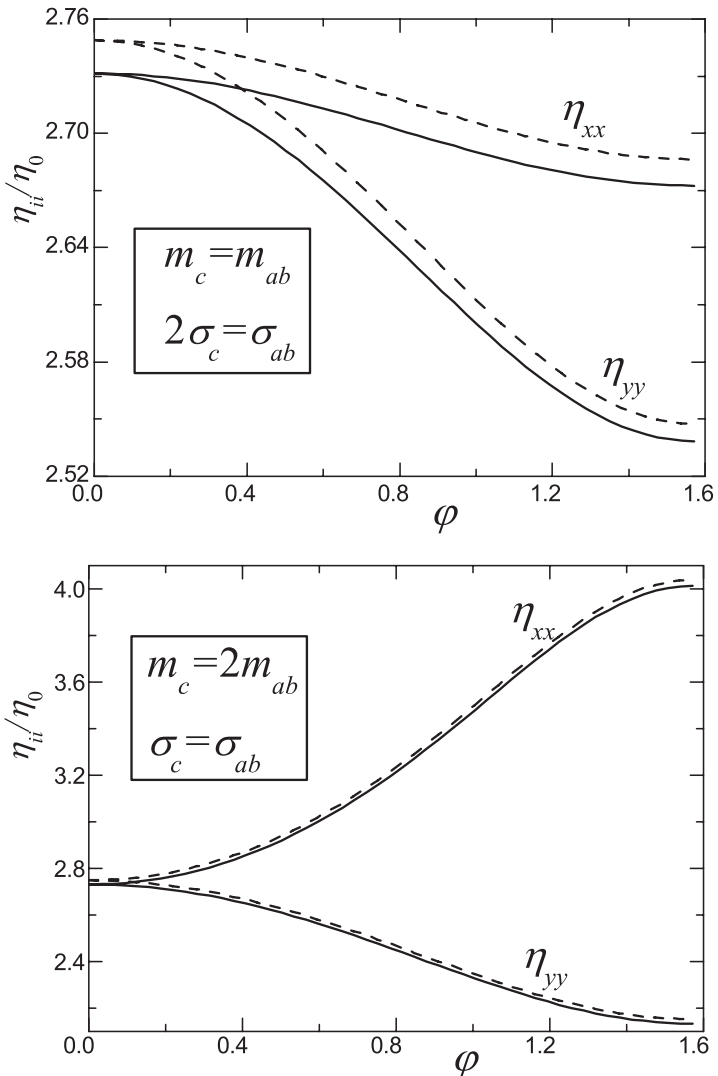

FIG. 2. The $\varphi$ dependencies of the viscosity components. Solid lines correspond to analytical results [Eqs. (45) and (46)], dashed lines show the results of numerical simulations. Here, $\eta_{x x}$ and $\eta_{y y}$ are measured in the units $\eta_{0}=\hbar \gamma|a| / b, \xi_{a b} / l_{E a b}=\sqrt{12}$.

These analytical expressions are in a good agreement with the asymptotics derived by numerical calculations:

$$
\begin{aligned}
& \eta_{x}=2 \frac{|a|}{b} \gamma \hbar \frac{1.58}{u}+O\left(u^{-2}\right), \\
& \eta_{y}=2 \frac{|a|}{b} \gamma \hbar \frac{0.86}{u}+O\left(u^{-2}\right) .
\end{aligned}
$$

When $s \gg u^{2}$, Eqs. (45) and (46) give

$$
\eta_{x} \approx \eta_{y} \approx 2 \pi \hbar \gamma \frac{|a|}{b} \ln \frac{\sqrt{s}}{u},
$$

which coincides with the main logarithmic term in Eqs. (33) and (34). One can see that the agreement between the exact and approximate asymptotics is quite good. This is a strong argument in favor of the applicability of Eqs. (45) and (46) for intermediate values of $s$ and $u$.

In Fig. 2, we plot the analytical and numerical $\varphi$ dependencies of the diagonal components of the full viscous drag tensor $\left(\hat{\eta}=\hat{\eta}_{p 0}+\hat{\eta}_{o h}\right)$.

\section{TEMPERATURE DEPENDENCE OF THE VISCOSITY ANISOTROPY}

Within the framework of the TDGL equation (4), the viscosity anisotropy $\eta_{x x} / \eta_{y y}$ does not depend on temperature. However, the region of applicability of Eq. (4) is limited by gapless superconductivity. In this section, we consider a more general approach based on the generalized TDGL equations ${ }^{19}$ 
(see also Ref. 12 for review):

$$
\begin{gathered}
2 \hbar \gamma \sqrt{1+q|\psi|^{2} /\left|\psi_{\infty}\right|^{2}} \frac{\partial|\psi|}{\partial t}=-\frac{\delta F}{\delta|\psi|} \\
\frac{\gamma|\psi|^{2}}{\sqrt{1+q|\psi|^{2} /\left|\psi_{\infty}\right|^{2}}}\left(\hbar \frac{\partial \theta}{\partial t}+2 e \Phi\right)=\frac{\hbar^{2}}{2} \nabla\left(|\psi|^{2} \hat{m}^{-1} \nabla \theta\right) \\
q=\frac{32 \pi^{2} \tau_{\mathrm{ph}}^{2} T_{c}\left(T_{c}-T\right)}{7 \zeta(3) \hbar^{2}}, \quad\left|\psi_{\infty}\right|^{2}=\frac{|a|}{b}
\end{gathered}
$$

Here, $T_{c}$ is the critical temperature and $\tau_{\mathrm{ph}}$ is the electronphonon mean free time. In the isotropic case, Eqs. (48) and (49) are valid for dirty superconductors when the temperature is close to $T_{c}$ and variations of the order parameter in space and in time are sufficiently slow.

The main relations for the viscous drag tensor can be derived in same way as described in Sec. II. As a result, we find that the viscosity still comprises two terms representing two mechanisms of dissipation, but the viscosity components undergo some changes. For example, Eq. (A19) is modified as follows:

$$
\left(\eta_{p 0}^{\prime}\right)_{i j}=2 \pi \hbar \gamma \frac{|a|}{b} \delta_{i j} \int_{0}^{\infty}\left(\frac{d f}{d \rho}\right)^{2} \rho \sqrt{1+q f^{2}(\rho)} d \rho .
$$

In order to obtain the counterparts of Eqs. (7), (8), (14), and (15), one should make the following substitutions in these equations:

$$
\begin{aligned}
u^{2} & \rightarrow \frac{u^{2}}{\sqrt{1+q}}, \\
f^{2} & \rightarrow \frac{\sqrt{1+q} f^{2}}{\sqrt{1+q f^{2}}}, \\
\eta_{i} & \rightarrow \quad \eta_{i} \sqrt{1+q}, \quad i=x, y .
\end{aligned}
$$

It can be seen from Eq. (49) that the electric field penetration depth is increased by a factor $(1+q)^{1 / 4}$ as compared to Eq. (12). It may seem that at low temperatures we would reach the $l_{E} \gg \xi$ limit, which has been analyzed in Ref. 9. However, this is not quite true because of the different relative impacts of the two mentioned mechanisms of dissipation in the simple and generalized TDGL models. Within the simple TDGL theory, the Bardeen-Stephen contribution and the relaxational term are of the same order of magnitude in the $l_{E} \gg \xi$ limit. On the contrary, in the generalized model, the viscosity is dominated by the relaxational term at low temperatures (see the following).

It is obvious that all main relations from Sec. III can be derived again within the generalized TDGL theory, but they are slightly modified. For example, Eq. (29) now reads as

$$
\eta_{x}=\eta_{y} \approx 2 \pi \hbar \gamma \frac{|a|}{b}\left[\frac{\sqrt{k_{2}}}{u}-\frac{1+2 q}{8 u^{2}}\right] .
$$

Now, consider the temperature dependence of the viscous drag tensor. The quantity $q$ depends on the temperature $T$, and $q_{T}^{\prime}<0$. Hence,

$$
\frac{\partial}{\partial T} \frac{\left(\eta_{p 0}\right)_{x x}}{\eta_{0}}=\frac{\partial}{\partial T} \frac{\left(\eta_{p 0}\right)_{y y}}{\eta_{0}}<0,
$$

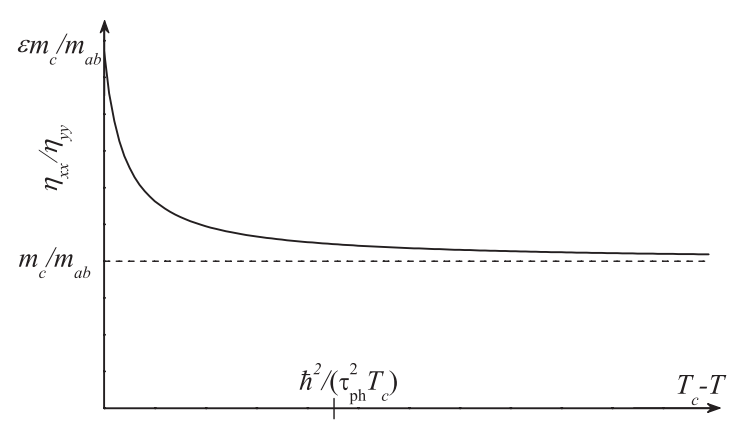

FIG. 3. Schematic temperature dependence of the viscosity anisotropy for $\varphi=\pi / 2$. The parameter $\epsilon$ is of the order of unity.

where $\eta_{0}=\hbar \gamma|a| / b$. On the other hand, the modified Eq. (38) can be written in the form

$$
\begin{aligned}
\tilde{\eta}\left(s_{x}, s_{y}, u\right)= & 2 \frac{|a|}{b} \gamma \hbar u^{2} \min _{\phi} \int\left[s_{x}\left(\frac{\partial \phi}{\partial x}\right)^{2}\right. \\
& \left.+s_{y}\left(\frac{\partial \phi}{\partial y}\right)^{2}+\frac{f^{2}(\rho)}{u^{2} \sqrt{1+q f^{2}}}\left(u^{2} \phi-\frac{y}{\rho^{2}}\right)^{2}\right] d x d y,
\end{aligned}
$$

if we leave Eqs. (37) unchanged. Hence,

$$
\frac{\partial}{\partial T} \frac{\left(\eta_{\mathrm{oh}}\right)_{x x}}{\eta_{0}}>0, \quad \frac{\partial}{\partial T} \frac{\left(\eta_{\mathrm{oh}}\right)_{y y}}{\eta_{0}}>0 .
$$

At sufficiently low temperatures, when $q \gg 1, s \lesssim 1$, and $u \sim$ 1 , it may happen that

$$
\left(\eta_{p 0}\right)_{x x} \gg\left(\eta_{\mathrm{oh}}\right)_{x x}, \quad\left(\eta_{p 0}\right)_{y y} \gg\left(\eta_{\mathrm{oh}}\right)_{y y} .
$$

Then, the viscosity anisotropy is determined by the relaxational term

$$
\frac{\eta_{x x}}{\eta_{y y}} \approx \frac{\left(\eta_{p 0}\right)_{x x}}{\left(\eta_{p 0}\right)_{y y}}=\frac{1+\mu}{1+\mu \cos ^{2} \varphi} .
$$

Note that when $s \neq 1, \eta_{x} \neq \eta_{y}$, so

$$
\frac{\eta_{x x}}{\eta_{y y}} \neq \frac{1+\mu}{1+\mu \cos ^{2} \varphi}
$$

when $q \lesssim 1$. We have proved that within the generalized TDGL theory, the viscosity anisotropy and the flux-flow conductivity anisotropy do depend on temperature. The schematic $T$ dependence of the ratio $\eta_{x x} / \eta_{y y}$ is plotted in Fig. 3 .

Note that in preceding papers, a correct order of magnitude estimate for the ratio $\eta_{x x} / \eta_{y y}$ has been obtained using either a standard TDGL equation ${ }^{9,10}$ or within a generalized BardeenStephen model. ${ }^{11}$ Certainly, these approaches do not allow us to determine the temperature dependence of the viscosity anisotropy obtained above.

\section{CONCLUSION}

By solving the time-dependent Ginzburg-Landau equation, we analyzed the viscous flux flow in anisotropic superconductors. The Bardeen-Stephen contribution to the viscous drag tensor $\hat{\eta}$ has been calculated in the $l_{E} \ll \xi$ and $l_{E c} \gg \xi_{c}$ limits. We emphasize that in these calculations, we did not use any simplifying assumptions concerning the shape of the order parameter in a static vortex. We suggested a variational 
procedure, which allowed us to derive the relations (45) and (46) suitable for arbitrary electric field penetration lengths $\left(l_{E a b}\right.$ and $\left.l_{E c}\right)$, coherence lengths $\left(\xi_{a b}\right.$ and $\left.\xi_{c}\right)$, and orientation of the magnetic field. Our results may be useful for interpretation of experimental data on flux-flow conductivity in isotropic and anisotropic superconductors in weak magnetic fields $\left(B \ll H_{c 2}\right)$.

Viscous flux flow has also been examined within a generalized TDGL theory. We found that the viscosity anisotropy may depend on temperature and, thus, the flux-flow conductivity anisotropy may be altered by heating or cooling the sample.

\section{ACKNOWLEDGMENTS}

This work was supported, in part, by the Russian Foundation for Basic Research, Russian Agency of Education under the Federal Program "Scientific and educational personnel of innovative Russia in 2009-2013," and European IRSES program SIMTECH (Contract No. 246937). We are thankful to N. B. Kopnin and J. R. Clem for helpful discussions.

\section{APPENDIX A}

In this Appendix, we derive Eqs. (7), (8), (14), and (15). In Eq. (4), it is convenient to make a scaling of the variables $x^{\prime}=x\left[m(\varphi) / m_{a b}\right]^{1 / 2}, y^{\prime}=y, z^{\prime}=z$. We rewrite Eq. (4) in the form

$$
\begin{gathered}
\gamma \hbar \frac{\partial|\psi|}{\partial t}=\frac{\hbar^{2}}{2 m_{a b}}\left[\nabla^{\prime 2}|\psi|-|\psi|\left(\nabla^{\prime} \theta\right)^{2}\right]-a|\psi|-b|\psi|^{3}, \\
\gamma|\psi|^{2}\left(\hbar \frac{\partial \theta}{\partial t}+2 e \Phi\right)=\frac{\hbar^{2}}{2 m_{a b}} \nabla^{\prime}\left(|\psi|^{2} \nabla^{\prime} \theta\right) .
\end{gathered}
$$

According to Eq. (5), the two-dimensional current $\mathbf{j}^{\prime}=$ $\left(j_{x}\left[m(\varphi) / m_{a b}\right]^{1 / 2}, j_{y}\right)$ satisfies the relation

$$
\operatorname{div}^{\prime} \mathbf{j}^{\prime}=0
$$

It follows from Eqs. (A2) and (A3) that

$$
\gamma|\psi|^{2}\left(\hbar \frac{\partial \theta}{\partial t}+2 e \Phi\right)=\frac{\hbar}{4 e} \nabla^{\prime}\left(\tilde{\sigma}_{n} \nabla^{\prime} \Phi\right)
$$

where we introduced the tensor $\tilde{\sigma}_{n}$ with components

$$
\begin{aligned}
& \tilde{\sigma}_{n x^{\prime} x^{\prime}}=s(\varphi) \sigma_{a b}, \quad \tilde{\sigma}_{n y^{\prime} y^{\prime}}=\sigma_{a b}, \\
& \tilde{\sigma}_{n x^{\prime} y^{\prime}}=\tilde{\sigma}_{n y^{\prime} x^{\prime}}=0,
\end{aligned}
$$

with $s(\varphi)$ given by Eq. (16). For a moving vortex, one should search the solution of Eqs. (A1), (A2), and (A3) in the form $\psi=\psi\left(\rho^{\prime}-\tilde{\mathbf{V}}_{L} t\right), \Phi=\Phi\left(\rho^{\prime}-\tilde{\mathbf{V}}_{L} t\right)$, where $\tilde{\mathbf{V}}_{\mathbf{L}}=$ $\left(V_{L x}\left[m(\varphi) / m_{a b}\right]^{1 / 2}, V_{L y}\right)$ and $\rho^{\prime}=\left(x^{\prime}, y^{\prime}\right)$. We expand $|\psi|$ and $\theta$ in powers of $V_{L}$ up to the first-order term, assuming the vortex velocity to be sufficiently small:

$$
\begin{aligned}
|\psi| & \approx \psi_{0}\left(\boldsymbol{\rho}^{\prime}-\tilde{\mathbf{V}}_{L} t\right)+\psi_{1}\left(\boldsymbol{\rho}^{\prime}-\tilde{\mathbf{V}}_{L} t\right) \\
\theta & \approx \theta_{0}\left(\boldsymbol{\rho}^{\prime}-\tilde{\mathbf{V}}_{L} t\right)+\theta_{1}\left(\boldsymbol{\rho}^{\prime}-\tilde{\mathbf{V}}_{L} t\right)
\end{aligned}
$$

Here, $\psi_{0}\left(\boldsymbol{\rho}^{\prime}\right)$ and $\theta_{0}\left(\boldsymbol{\rho}^{\prime}\right)$ correspond to a static vortex. The functions $\psi_{1}, \theta_{1}$, and $\Phi$ are of the order $V_{L}$. We substitute
(A6) and (A7) into Eqs. (A1), (A3), and (A4):

$$
\begin{gathered}
-a \psi_{0}-b \psi_{0}^{3}+\frac{\hbar^{2}}{2 m_{a b}}\left[\nabla^{\prime 2} \psi_{0}-\psi_{0}\left(\nabla^{\prime} \theta_{0}\right)^{2}\right]=0 \\
\frac{\hbar^{2}}{2 m_{a b}}\left[\nabla^{\prime 2} \psi_{1}-\psi_{1}\left(\nabla^{\prime} \theta_{0}\right)^{2}-2 \psi_{0} \nabla^{\prime} \theta_{0} \cdot \nabla^{\prime} \theta_{1}\right] \\
-a \psi_{1}-3 b \psi_{0}^{2} \psi_{1}=-\gamma \hbar\left(\tilde{\mathbf{V}}_{L} \nabla^{\prime}\right) \psi_{0} \\
\operatorname{div}^{\prime} \mathbf{j}_{1}^{\prime}=0, \quad \mathbf{j}_{0}^{\prime}=\frac{2 e \hbar \psi_{0}^{2}}{m_{a b}} \nabla^{\prime} \theta_{0}, \\
\mathbf{j}_{1}^{\prime}=\frac{2 e \hbar}{m_{a b}}\left(2 \psi_{0} \psi_{1} \nabla^{\prime} \theta_{0}+\psi_{0}^{2} \nabla^{\prime} \theta_{1}\right)-\tilde{\sigma}_{n} \nabla^{\prime} \Phi \\
\frac{\hbar \sigma_{a b}}{4 e}\left[s(\varphi) \frac{\partial^{2} \Phi}{\partial x^{\prime 2}}+\frac{\partial^{2} \Phi}{\partial y^{\prime 2}}\right]=\gamma \psi_{0}^{2}\left(2 e \Phi-\hbar \tilde{\mathbf{V}}_{\mathbf{L}} \cdot \nabla^{\prime} \theta_{0}\right) .
\end{gathered}
$$

Now, we introduce some new notations: $\psi_{d}=\left(\mathbf{d} \nabla^{\prime}\right) \psi_{0}, \theta_{d}=$ $\left(\mathbf{d} \nabla^{\prime}\right) \theta_{0}, \mathbf{j}_{d}^{\prime}=\left(\mathbf{d} \nabla^{\prime}\right) \mathbf{j}_{0}^{\prime}$, where $\mathbf{d}$ is an arbitrary vector. A simple equation connecting $\psi_{d}$ and $\theta_{d}$ can be obtained by applying the operator $\mathbf{d} \nabla^{\prime}$ to Eq. (A8):

$$
\begin{aligned}
& \frac{\hbar^{2}}{2 m_{a b}}\left[\nabla^{\prime 2} \psi_{d}-\psi_{d}\left(\nabla^{\prime} \theta_{0}\right)^{2}-2 \psi_{0} \nabla^{\prime} \theta_{0} \cdot \nabla^{\prime} \theta_{d}\right] \\
& \quad-a \psi_{d}-3 b \psi_{0}^{2} \psi_{d}=0 .
\end{aligned}
$$

The vector $\mathbf{j}_{d}^{\prime}$ satisfies the obvious relation $\operatorname{div}^{\prime} \mathbf{j}_{d}^{\prime}=0$. Let us multiply Eq. (A9) by $\psi_{d}$, subtract Eq. (A13) multiplied by $\psi_{1}$, and integrate the resulting equation over a large volume containing the whole vortex. After some simple algebra and integration by parts, we obtain

$$
\begin{aligned}
& -\gamma \hbar \int\left(\tilde{\mathbf{V}}_{L} \nabla^{\prime}\right) \psi_{0} \psi_{d} d^{3} \mathbf{r}^{\prime} \\
& =\frac{\hbar}{4 e} \int\left[\left(\mathbf{j}_{1}^{\prime}+\tilde{\sigma}_{n} \nabla^{\prime} \Phi\right) \nabla^{\prime} \theta_{d}-\mathbf{j}_{d}^{\prime} \nabla^{\prime} \theta_{1}\right] d^{3} \mathbf{r}^{\prime} \\
& =\frac{\hbar}{4 e} \int\left(\tilde{\sigma}_{n} \nabla^{\prime} \Phi\right) \nabla^{\prime} \theta_{d} d^{3} \mathbf{r}^{\prime}+\frac{\hbar}{4 e} \int_{S}\left(\mathbf{j}_{1}^{\prime} \theta_{d}-\mathbf{j}_{d}^{\prime} \theta_{1}\right) d \mathbf{S} .
\end{aligned}
$$

Here, $S$ is a surface far from the vortex axis. At large distances $\rho^{\prime} \gg \xi_{a b}$ we have

$$
\mathbf{j}_{1}^{\prime} \approx \frac{2 e \hbar|a|}{b m_{a b}} \nabla^{\prime} \theta_{1}=\mathbf{j}_{\mathrm{tr}}^{\prime}, \quad \theta_{1}=\frac{b m_{a b}}{2 e \hbar|a|}\left(\mathbf{j}_{\mathrm{tr}}^{\prime} \cdot \boldsymbol{\rho}^{\prime}\right)+\text { const },
$$

where $\mathbf{j}_{\mathrm{tr}}^{\prime}$ is the transport current which is constant. If we calculate the surface integral in Eq. (A14) and make some simple transformations, we obtain the force balance equation ${ }^{9}$

$$
\begin{gathered}
\frac{\pi \hbar}{e}\left[\mathbf{d} \cdot\left(\mathbf{j}_{\mathrm{tr}}^{\prime} \times \mathbf{n}\right)\right]=-2 \pi \gamma \hbar\left(\mathbf{d} \cdot \tilde{\mathbf{V}}_{L}\right) \int_{0}^{\infty}\left(\frac{d \psi_{0}}{d \rho}\right)^{2} \rho d \rho \\
+\frac{\hbar \sigma_{a b}}{2 e} \int\left[s(\varphi) \frac{\partial^{2} \Phi}{\partial x^{\prime 2}}+\frac{\partial^{2} \Phi}{\partial y^{\prime 2}}\right]\left(\mathbf{d} \cdot \nabla^{\prime} \theta_{0}\right) d^{2} \boldsymbol{\rho}^{\prime}, \quad(\mathrm{A} 15)
\end{gathered}
$$

where $\mathbf{n}$ is a unit vector along the magnetic field. If we compare Eqs. (1) and (A15), we can see that the viscous drag tensor in 
the frame $\left(x^{\prime}, y^{\prime}, z^{\prime}\right)$ should be defined as follows:

$$
\begin{aligned}
& \mathbf{d} \cdot \hat{\eta}^{\prime} \tilde{\mathbf{V}}_{L}=2 \pi \gamma \hbar\left(\mathbf{d} \cdot \tilde{\mathbf{V}}_{L}\right) \frac{|a|}{b} \int_{0}^{\infty}\left(\frac{d f}{d \rho}\right)^{2} \rho d \rho \\
& -\frac{\hbar \sigma_{a b}}{2 e} \int\left[s(\varphi) \frac{\partial^{2} \Phi}{\partial x^{\prime 2}}+\frac{\partial^{2} \Phi}{\partial y^{\prime 2}}\right]\left(\mathbf{d} \cdot \nabla^{\prime} \theta_{0}\right) d^{2} \rho^{\prime},
\end{aligned}
$$

where we introduced the function

$$
f(\rho)=\psi_{0}\left(\rho \xi_{a b}\right) \sqrt{\frac{b}{|a|}} .
$$

The components of the tensor $\hat{\eta}$ in the frame $(x, y, z)$ are given by

$$
\eta_{x x}=\left[m(\varphi) / m_{a b}\right]^{1 / 2} \eta_{x^{\prime} x^{\prime}}^{\prime}, \quad \eta_{y y}=\left[m_{a b} / m(\varphi)\right]^{1 / 2} \eta_{y^{\prime} y^{\prime}}^{\prime} .
$$

The right-hand side of Eq. (A16) contains two terms, representing two mechanisms of dissipation. The viscous drag tensor due to relaxation of the order parameter is ${ }^{6-9}$

$$
\begin{gathered}
\left(\eta_{p 0}^{\prime}\right)_{i j}=2 \pi \hbar \gamma \frac{|a|}{b} \alpha_{1} \delta_{i j}, \\
\alpha_{1}=\int_{0}^{\infty}\left(\frac{d f}{d \rho}\right)^{2} \rho d \rho=0.279 .
\end{gathered}
$$

The second term in the right-hand side of Eq. (A16) defines the Ohmic viscosity tensor $\hat{\eta}_{o h}^{\prime}$, which is to be evaluated:

$$
\mathbf{d} \cdot \hat{\eta}_{o h}^{\prime} \tilde{\mathbf{V}}_{L}=-\frac{\hbar \sigma_{a b}}{2 e} \int\left[s(\varphi) \frac{\partial^{2} \Phi}{\partial x^{\prime 2}}+\frac{\partial^{2} \Phi}{\partial y^{\prime 2}}\right]\left(\mathbf{d} \nabla^{\prime} \theta_{0}\right) d^{2} \boldsymbol{\rho}^{\prime}
$$

Now, if we substitute $\Phi$ in the form (17) into Eqs. (A12) and (A20) and switch to the coordinates $\left(x_{1}, y_{1}\right)$ [see Eq. (11)], we obtain Eqs. (7), (8), (14), and (15).

\section{APPENDIX B}

In this Appendix, we will derive Eq. (24). First, we divide the integral in Eq. (7) into two parts:

$$
\begin{gathered}
\eta_{x}=\eta_{x 1}+\eta_{x 2}, \\
\eta_{x 1}=-2 \frac{|a|}{b} \gamma \hbar \int_{\rho_{1}<\rho_{0} / \sqrt{u}} f^{2}\left(\rho_{1}\right) \frac{y_{1}}{\rho_{1}^{2}}\left(u^{2} \Phi_{x}-\frac{y_{1}}{\rho_{1}^{2}}\right) d x_{1} d y_{1}, \\
\eta_{x 2}=-2 \frac{|a|}{b} \gamma \hbar \int_{\rho_{1}>\rho_{0} / \sqrt{u}}^{f^{2}\left(\rho_{1}\right)} \frac{y_{1}}{\rho_{1}^{2}}\left(u^{2} \Phi_{x}-\frac{y_{1}}{\rho_{1}^{2}}\right) d x_{1} d y_{1},
\end{gathered}
$$

where $\rho_{0}=u^{1 / 6+\delta}, \delta \ll 1 / 6$. Note that the left-hand side of Eq. (14) is small when $\rho_{1}>\rho_{0} / \sqrt{u} \gg u^{-1 / 2}$, so it can be accounted for by perturbation theory:

$$
\begin{gathered}
\Phi_{x}=\frac{y_{1}}{u^{2} \rho_{1}^{2}}+\Phi_{x 1}+\Phi_{x 2}+\ldots, \\
\Phi_{x 1}=\frac{1}{u^{4} f^{2}\left(\rho_{1}\right)}\left(\frac{\partial^{2}}{\partial y_{1}^{2}}+s \frac{\partial^{2}}{\partial x_{1}^{2}}\right) \frac{y_{1}}{\rho_{1}^{2}}, \\
\Phi_{x 2}=\frac{1}{u^{6}}\left[\frac{1}{f^{2}\left(\rho_{1}\right)}\left(\frac{\partial^{2}}{\partial y_{1}^{2}}+s \frac{\partial^{2}}{\partial x_{1}^{2}}\right)\right]^{2} \frac{y_{1}}{\rho_{1}^{2}} .
\end{gathered}
$$

The main contribution to the integral in Eq. (B3) is determined by small $\rho_{1}$. The integral of $\Phi_{x 2}$ is of the order of $\left(u \rho_{0}^{6}\right)^{-1} \ll$ $u^{-2}$; the integrals of higher-order terms are also negligibly small, hence,

$$
\begin{gathered}
\eta_{x 2} \approx-2 \frac{|a|}{b} \gamma \hbar \frac{I_{0 x}^{\prime}}{u} \\
I_{0 x}^{\prime}=\int_{\rho>\rho_{0}} \frac{y}{\rho^{2}}\left(\frac{\partial^{2}}{\partial y^{2}}+s \frac{\partial^{2}}{\partial x^{2}}\right) \frac{y}{\rho^{2}} d x d y .
\end{gathered}
$$

Let us consider the component $\eta_{x 1}$. In the new variables introduced in Sec. III A, Eq. (B2) reads as

$$
\eta_{x 1}=-2 \frac{|a|}{b} \gamma \hbar \int_{\rho<\rho_{0}} f^{2}\left(\frac{\rho}{\sqrt{u}}\right) \frac{y}{\rho^{2}}\left(\tilde{\Phi}_{x}-\frac{y}{\rho^{2}}\right) d x d y .
$$

Now, we estimate the term $R_{x}$ introduced in Eq. (21). It satisfies the following relation:

$$
\begin{aligned}
\frac{\partial^{2} R_{x}}{\partial y^{2}}+s \frac{\partial^{2} R_{x}}{\partial x^{2}}-u f^{2}\left(\frac{\rho}{\sqrt{u}}\right) R_{x} \\
=\left[u f^{2}\left(\frac{\rho}{\sqrt{u}}\right)-k_{2} \rho^{2}-\frac{k_{4} \rho^{4}}{u}\right]\left(\Phi_{x}^{(0)}-\frac{y}{\rho^{2}}\right) \\
+\left[u f^{2}\left(\frac{\rho}{\sqrt{u}}\right)-k_{2} \rho^{2}\right] \frac{\Phi_{x}^{(1)}}{u} .
\end{aligned}
$$

Note that when $\rho \ll \sqrt{u}$, the source in the right-hand side of (B7) can be presented as $S(x, y) u^{-2}$, where $S(x, y)$ is some function independent of $u$. Since (B7) is a screening equation, the function $R_{x}(x, y, u)$ for small $\rho$ does not depend on the behavior of the source in the area of big $\rho$ and can be presented as $R_{x}=\tilde{R}_{x}(x, y) u^{-2}$. On the other hand, when $\rho \gg 1$, the derivatives in the left-hand side of Eq. (B7) are small, hence, in the area $1 \ll \rho \ll \sqrt{u}$,

$$
\begin{gathered}
R_{x} \approx \frac{1}{u^{2}}\left[-\frac{k_{6} \rho^{4}}{k_{2}}\left(\Phi_{x}^{(0)}-\frac{y}{\rho^{2}}\right)-\frac{k_{4} \rho^{2}}{k_{2}} \Phi_{x}^{(1)}\right], \\
\left|R_{x}\right| \leqslant \frac{\text { const }}{\rho u^{2}} .
\end{gathered}
$$

Now, we substitute $\tilde{\Phi}_{x}$ in the form (21) into (B6):

$$
\eta_{x 1}=-2 \frac{|a|}{b} \gamma \hbar\left(\frac{I_{1 x}^{\prime}}{u}+\frac{I_{2 x}^{\prime}}{u^{2}}+I_{3 x}\right),
$$

where

$$
\begin{gathered}
I_{1 x}^{\prime}=\int_{\rho<\rho_{0}} k_{2} y\left(\Phi_{x}^{(0)}-\frac{y}{\rho^{2}}\right) d x d y, \quad \text { (B10) } \\
I_{2 x}^{\prime}=\int_{\rho<\rho_{0}} \frac{y}{\rho^{2}}\left[k_{4} \rho^{4}\left(\Phi_{x}^{(0)}-\frac{y}{\rho^{2}}\right)+k_{2} \rho^{2} \Phi_{x}^{(1)}\right] d x d y, \\
I_{3 x}=\int_{\rho<\rho_{0}}\left[f^{2}\left(\frac{\rho}{\sqrt{u}}\right)-\frac{k_{2} \rho^{2}}{u}-\frac{k_{4} \rho^{4}}{u^{2}}\right]\left(\Phi_{x}^{(0)}-\frac{y}{\rho^{2}}\right) \frac{y}{\rho^{2}} d x d y \\
+\int_{\rho<\rho_{0}}\left[f^{2}\left(\frac{\rho}{\sqrt{u}}\right)-\frac{k_{2} \rho^{2}}{u}\right] \frac{\Phi_{x}^{(1)}}{u} \frac{y}{\rho^{2}} d x d y \\
+\int_{\rho<\rho_{0}} f^{2}\left(\frac{\rho}{\sqrt{u}}\right) R_{x} \frac{y}{\rho^{2}} d x d y .
\end{gathered}
$$


One can easily prove that

$$
\begin{gathered}
\Phi_{x}^{(0)}=\frac{y}{\rho^{2}}+\frac{1}{k_{2} \rho^{2}}\left(\frac{\partial^{2}}{\partial y^{2}}+s \frac{\partial^{2}}{\partial x^{2}}\right) \frac{y}{\rho^{2}}+O\left(\rho^{-9}\right), \\
k_{4} \rho^{4}\left(\Phi_{x}^{(0)}-\frac{y}{\rho^{2}}\right)+k_{2} \rho^{2} \Phi_{x}^{(1)}=O\left(\rho^{-5}\right) .
\end{gathered}
$$

From Eqs. (B13), (B14), and (B8), we can see that all integrals in Eq. (B12) are of the order $\rho_{0}^{2} / u^{3}$. Thus, $\left|I_{3 x}\right| \ll u^{-2}$, so it can be neglected. Also, we can integrate in Eqs. (B10) and (B11) over the whole $x y$ plane since

$$
\begin{gathered}
\left|I_{0 x}^{\prime}+I_{1 x}^{\prime}-I_{1 x}(s)\right| \ll u^{-1}, \\
\left|I_{2 x}^{\prime}-I_{2 x}(s)\right| \ll 1,
\end{gathered}
$$

Finally, taking into account Eqs. (B1), (B5), and (B9), we obtain Eq. (24).

\section{APPENDIX C}

In this Appendix, we consider in detail the derivation of Eqs. (33) and (34). We will present here the calculations for the $\eta_{y}$ component since the calculations for the $\eta_{x}$ component are less complicated. First, we rewrite Eq. (15) in the form

$$
\begin{aligned}
& s \frac{\partial^{2} \Phi_{y}}{\partial x^{2}}-u^{2} \Phi_{y}+\frac{x}{\rho^{2}} f^{2}(\rho) \\
& \quad=-\frac{\partial^{2} \Phi_{y}}{\partial y^{2}}-u^{2} \Phi_{y}\left[1-f^{2}(\rho)\right] .
\end{aligned}
$$

The index " 1 " is omitted. It will be proved below that the terms in the right-hand side of Eq. $(\mathrm{C} 1)$ give a small contribution to the viscosity, so they can be neglected. Then, the solution of Eq. (C1) has the form

$$
\Phi_{y} \approx \Phi_{y 0}=\int_{-\infty}^{+\infty} \frac{x^{\prime} f^{2}\left(\rho^{\prime}\right)}{x^{\prime 2}+y^{2}} \frac{\exp \left(-u\left|x-x^{\prime}\right| / \sqrt{s}\right)}{2 u \sqrt{s}} d x^{\prime}
$$

where $\rho^{\prime}=\sqrt{x^{\prime 2}+y^{2}}$. Consider a quantity $y_{0}$ in the range $1 \ll y_{0} \ll \sqrt{s} / u$ (for example, $y_{0}=s^{1 / 4} / u^{1 / 2}$ ). We divide the integral in Eq. (8) into three parts:

$$
\begin{aligned}
\eta_{y}= & -2 \frac{|a|}{b} \gamma \hbar\left[\int_{|y|<y_{0}} f^{2}(\rho) \frac{x}{\rho^{2}} u^{2} \Phi_{y} d x d y\right. \\
& \left.-\int_{|y|<y_{0}} f^{2}(\rho) \frac{x^{2}}{\rho^{4}} d x d y\right] \\
& -4 \frac{|a|}{b} \gamma \hbar \int_{y>y_{0}} f^{2}(\rho) \frac{x}{\rho^{2}}\left(u^{2} \Phi_{y}-\frac{x}{\rho^{2}}\right) d x d y .
\end{aligned}
$$

Using the inequality

$$
f^{2}(\rho)<\frac{\rho^{2}}{A_{1}+\rho^{2}},
$$

where $A_{1}$ is some constant, we can estimate the first integral

$$
\left|\int_{|y|<y_{0}} f^{2}(\rho) \frac{x}{\rho^{2}} u^{2} \Phi_{y} d x d y\right| \leqslant \operatorname{const} \frac{y_{0} u}{\sqrt{s}}\left(\ln \frac{\sqrt{s}}{u}\right)^{2} \ll 1 \text {. }
$$

Here and further, "const" denotes a constant independent of any parameters. The second term in Eq. (C3) has the following asymptotics when $y_{0} \gg 1$ :

$$
\int_{|y|<y_{0}} f^{2}(\rho) \frac{x^{2}}{\rho^{4}} d x d y \approx \pi \ln y_{0}+C_{y} .
$$

The constant $C_{y}$ will be evaluated below. The third integral in Eq. (C3) can be simplified if we take into account that $\rho>y_{0}$, $\rho^{\prime}>y_{0}$, and $y_{0} \gg 1$, so we can substitute unity instead of $f^{2}$.

$$
\begin{aligned}
& \int_{y>y_{0}} f^{2}(\rho) \frac{x}{\rho^{2}}\left(u^{2} \Phi_{y}-\frac{x}{\rho^{2}}\right) d x d y \\
& \approx \int_{y_{0}}^{\infty}\left(\frac{\pi u}{\sqrt{s}} \int_{-\infty}^{+\infty} \frac{y \exp \left(-\frac{u|x|}{\sqrt{s}}\right)}{x^{2}+4 y^{2}} d x-\frac{\pi}{2 y}\right) d y \\
& =\frac{\pi}{2} \int_{0}^{\infty} d x \int_{y_{0}}^{\infty} d y\left(\frac{4 y}{s x^{2} / u^{2}+4 y^{2}}-\frac{1}{y}\right) e^{-x} \\
& \approx-\frac{\pi}{4} \int_{0}^{\infty} \ln \frac{s x^{2}}{4 y_{0}^{2} u^{2}} e^{-x} d x=\frac{\pi}{2} \ln y_{0}-\frac{\pi}{4} \ln \frac{s}{4 u^{2}}+\frac{\pi}{2} \mathcal{C},
\end{aligned}
$$

where $\mathcal{C}$ is the Euler constant:

$$
\mathcal{C}=-\int_{0}^{\infty} \ln x e^{-x} d x \approx 0.577
$$

Using (C3)-(C7), we obtain

$$
\eta_{y}=2 \pi \hbar \gamma \frac{|a|}{b}\left(\frac{1}{2} \ln \frac{s}{4 u^{2}}+\frac{C_{y}}{\pi}-\mathcal{C}\right) .
$$

The component $\eta_{x}$ can be calculated in a similar way:

$$
\begin{array}{r}
\eta_{x}=2 \pi \hbar \gamma \frac{|a|}{b}\left(\frac{1}{2} \ln \frac{s}{4 u^{2}}+\frac{C_{x}}{\pi}-\mathcal{C}\right), \\
C_{x}=\lim _{y_{0} \rightarrow \infty}\left(\int_{|y|<y_{0}} f^{2}(\rho) \frac{y^{2}}{\rho^{4}} d x d y-\pi \ln y_{0}\right) .
\end{array}
$$

Now, we evaluate $C_{x}$ and $C_{y}$. Here, the constant $g_{4}^{\prime}$ from Ref. 7 will be useful:

$$
g_{4}^{\prime}=\int_{0}^{\infty}\left[f^{2}(\rho)-\frac{\rho^{2}}{1+\rho^{2}}\right] \rho^{-1} d \rho=-0.3982 .
$$

It is easy to check that

$$
C_{x}=\pi g_{4}^{\prime}+\lim _{y_{0} \rightarrow \infty} \int_{|y|<y_{0}, \rho>y_{0}} \underset{y^{2} \rho^{-4}}{ } d x d y=\pi\left(g_{4}^{\prime}+\ln 2-\frac{1}{2}\right) .
$$

Similarly,

$$
C_{y}=\pi\left(g_{4}^{\prime}+\ln 2+\frac{1}{2}\right) .
$$

Finally, the components of the viscous drag tensor take the form

$$
\begin{aligned}
& \eta_{x}=2 \pi \hbar \gamma \frac{|a|}{b}\left(\ln \frac{\sqrt{s}}{u}+g_{4}^{\prime}-\mathcal{C}-\frac{1}{2}\right), \\
& \eta_{y}=2 \pi \hbar \gamma \frac{|a|}{b}\left(\ln \frac{\sqrt{s}}{u}+g_{4}^{\prime}-\mathcal{C}+\frac{1}{2}\right) .
\end{aligned}
$$

If we substitute $\mathcal{C}$ and $g_{4}^{\prime}$ with their numerical values, we obtain Eqs. (33) and (34).

Now, it is necessary to prove our assumption concerning the right-hand side of Eq. (C1). Consider it as a perturbation. The first-order correction to the approximate solution $\Phi_{y 0}$ has 
the form

$$
\begin{gathered}
\Phi_{y 1}=R_{y}^{(1)}+R_{y}^{(2)} \\
R_{y}^{(1)}=\int_{-\infty}^{+\infty} \frac{u \Phi_{y 0}\left(x^{\prime}, y\right)\left[1-f^{2}\left(\rho^{\prime}\right)\right]}{2 \sqrt{s}} \exp \left(-\frac{u\left|x-x^{\prime}\right|}{\sqrt{s}}\right) d x^{\prime} \\
R_{y}^{(2)}=\frac{1}{2 u \sqrt{s}} \int_{-\infty}^{+\infty} \frac{\partial^{2} \Phi_{y 0}}{\partial y^{2}}\left(x^{\prime}, y\right) \exp \left(-\frac{u\left|x-x^{\prime}\right|}{\sqrt{s}}\right) d x^{\prime} .
\end{gathered}
$$

The contribution of $\Phi_{y 1}$ to $\eta_{y}$ is equal to

$$
\Delta \eta_{y}=-2 \frac{|a|}{b} \gamma \hbar\left(I_{y}^{(1)}+I_{y}^{(2)}\right),
$$

where

$$
\begin{aligned}
& I_{y}^{(1)}=\int f^{2}(\rho) \frac{x}{\rho^{2}} u^{2} R_{y}^{(1)} d x d y, \\
& I_{y}^{(2)}=\int f^{2}(\rho) \frac{x}{\rho^{2}} u^{2} R_{y}^{(2)} d x d y .
\end{aligned}
$$

We will show that $\left|I_{y}^{(1)}\right| \ll 1$ and $\left|I_{y}^{(2)}\right| \ll 1$ when $s \gg u^{2}$.

A simple estimate for $\left|\Phi_{y 0}\right|$ can be obtained with the help of (C4):

$$
\left|\Phi_{y 0}\right| \leqslant \operatorname{const} \frac{\ln \frac{\sqrt{s}}{u}}{u \sqrt{s}} .
$$

Using the inequality

$$
\frac{\rho^{2}}{A_{2}+\rho^{2}}<f^{2}(\rho)
$$

and Eq. (C18), we can estimate $I_{y}^{(1)}$ :

$$
\left|I_{y}^{(1)}\right| \leqslant \operatorname{const} \frac{u}{\sqrt{s}} \ln \frac{\sqrt{s}}{u} \ll 1 .
$$

For all $x^{\prime}$ and $y$, we can write

$$
\left|\frac{\partial^{2}}{\partial y^{2}}\left(\frac{1}{x^{\prime 2}+y^{2}} f^{2}\left(\rho^{\prime}\right)\right)\right| \leqslant \frac{\text { const }}{\left(A_{3}+x^{\prime 2}+y^{2}\right)^{2}},
$$

whence

$$
\begin{aligned}
\left|I_{y}^{(2)}\right| & \leqslant \frac{\text { const }}{s} \int \frac{|x| \exp \left(-\frac{u\left|x-x^{\prime}\right|}{\sqrt{s}}\right) \exp \left(-\frac{u\left|x^{\prime \prime}-x^{\prime}\right|}{\sqrt{s}}\right)}{\left(A_{3}+x^{\prime \prime 2}\right)\left(A_{1}+x^{2}\right)} d x^{\prime} d x^{\prime \prime} d x \\
& \leqslant \frac{\text { const }}{s} \ln \frac{\sqrt{s}}{u} \int \frac{\exp \left(-\frac{u\left|x^{\prime \prime}-x^{\prime}\right|}{\sqrt{s}}\right)}{x^{\prime \prime 2}+A_{3}} d x^{\prime} d x^{\prime \prime} \\
& \leqslant \frac{\text { const }}{\sqrt{s} u} \ln \frac{\sqrt{s}}{u} \ll 1
\end{aligned}
$$

when $u \gtrsim 1$.
${ }^{1}$ M. Tinkham, Phys. Rev. Lett. 13, 804 (1964).

${ }^{2}$ I. Bardeen and M. I. Stephen, Phys. Rev. 140, A1197 (1965).

${ }^{3}$ A. Schmid, Phys. Kondens. Mater. 5, 302 (1966).

${ }^{4}$ L. P. Gor'kov and N. B. Kopnin, ZhETF 60, 2331 (1971) [Sov. Phys. JETP 33, 1251 (1971)].

${ }^{5}$ L. P. Gor'kov and N. B. Kopnin, Usp. Fiz. Nauk 116, 413 (1975) [Sov. Phys. Usp. 18, 496 (1975)].

${ }^{6}$ C. R. Hu and R. S. Thompson, Phys. Rev. B 6, 110 (1972).

${ }^{7}$ C. R. Hu, Phys. Rev. B 6, 1756 (1972).

${ }^{8}$ M. Yu. Kupriyanov and K. K. Likharev, Pis'ma v ZhETF 15, 349 (1972) [JETP Lett. 15, 247 (1972)].

${ }^{9}$ V. M. Genkin and A. S. Mel'nikov, ZhETF 95, 2170 (1989) [Sov. Phys. JETP 68, 1254 (1989)].

${ }^{10}$ B. I. Ivlev and N. B. Kopnin, Europhys. Lett. 15, 349 (1991).

${ }^{11}$ Z. Hao and J. R. Clem, IEEE Trans. Magn. 27, 1086 (1991).

${ }^{12}$ N. B. Kopnin, Theory of Nonequilibrium Superconductivity (Oxford University Press, Oxford, 2001).

${ }^{13}$ K. Shirai, H. Kashiwaya, S. Miura, M. Ishikado, H. Eisaki, A. Iyo, I. Kurosawa, and S. Kashiwaya, Phys. C (Amsterdam) 470, 1473 (2010).

${ }^{14}$ P. M. Shirage, K. Miyazawa, M. Ishikado, K. Kihou, C. H. Lee, N. Takeshita, H. Matsuhata, R. Kumai, Y. Tomioka, T. Ito, H. Kito,
H. Eisaki, S. Shamoto, and A. Iyo, Phys. C (Amsterdam) 469, 355 (2009).

${ }^{15}$ N. Ni, S. L. Budko, A. Kreyssig, S. Nandi, G. E. Rustan, A. I. Goldman, S. Gupta, J. D. Corbett, A. Kracher, and P. C. Canfield, Phys. Rev. B 78, 014507 (2008).

${ }^{16}$ X. F. Wang, T. Wu, G. Wu, H. Chen, Y. L. Xie, J. J. Ying, Y. J. Yan, R. H. Liu, and X. H. Chen, Phys. Rev. Lett. 102, 117005 (2009).

${ }^{17}$ M. A. Tanatar, N. Ni, G. D. Samolyuk, S. L. Bud'ko, P. C. Canfield, and R. Prozorov, Phys. Rev. B 79, 134528 (2009).

${ }^{18}$ M. A. Tanatar, N. Ni, C. Martin, R. T. Gordon, H. Kim, V. G. Kogan, G. D. Samolyuk, S. L. Budko, P. C. Canfield, R. Prozorov, Phys. Rev. B 79, 094507 (2009).

${ }^{19}$ R. J. Watts-Tobin, Y. Krähenbühl, and L. Kramer, J. Low Temp. Phys. 42, 459 (1981).

${ }^{20}$ A. V. Chubukov, D. V. Efremov, and I. Eremin, Phys. Rev. B 78, 134512 (2008); Y. Bang, H.-Y. Choi, and H. Won, ibid. 79, 054529 (2009); A. B. Vorontsov, M. G. Vavilov, and A. V. Chubukov, ibid. 79, 140507 (2009); Y. Bang, Europhys. Lett. 86, 47001 (2009); A. Glatz and A. E. Koshelev, Phys. Rev. B 82, 012507.

${ }^{21}$ J. R. Clem, J. Low. Temp. Phys. 18, 427 (1975). 